\title{
Trends in the Global ICT \\ Industry-Globalization, Competition and the Internet of Things
}

\author{
Peter Nolan
}

\begin{abstract}
This essay provides an analysis of the Internet of Things. The facts provided differ greatly from the opinions that dominate media both inside and outside of China. The Internet of Things needs to be considered in terms of not just a single part of the architecture, but rather, in terms of the comprehensive structure of global data transmission, storage and analysis. Huawei has a significant role within one segment of that structure. However, within the whole structure, Huawei has a small role in the Internet of Things. So far, the entire structure of the Internet of Things is dominated by firms from high-income countries, especially the US. Three super-large firms-Amazon, Microsoft and Alphabet-Google-have leveraged their dominant position to establish an early lead in cloud computing software and services. These three behemoths account for $38 \%$ of the total R\&D spending and $34 \%$ of the net sales revenue for the 321 firms in the G2500 ICT software and services sector. Collectively, they account for almost $60 \%$ of global revenue from software services for the public cloud. Huawei is China's only high technology company with a significant global market share outside China.
\end{abstract}

Keywords The Internet of Things $\cdot$ Huawei has a small role in the Internet of Things $\cdot$ Amazon, Microsoft and Alphabet-Google-have leveraged their dominant position - Huawei is China's only high technology company with a significant global market

\section{Background}

Since the 1980s, a revolution has taken place in information and communication technology (ICT). The revolution has penetrated every sector of the economy and society. It has transformed the way in which governments function. It has transformed financial services. It has also transformed every part of non-financial services, including

The data in this paper are from P. Nolan, China and the West: Crossroads of Innovation, Routledge, 2022 (forthcoming).

P. Nolan (凶)

China Centre, Jesus College, Cambridge University, Cambridge, UK

(C) The Author(s) 2021

H. Wang and A. Michie (eds.), Consensus or Conflict?, China and Globalization,

https://doi.org/10.1007/978-981-16-5391-9_22 
telecommunications, retail, travel and tourism, entertainment, mass media, professional services, health care and education. It has transformed every part of the world's manufacturing system, including aerospace, automobiles, beverages and biomedical products. The revolution has transformed the internal operations of global companies, enabling them to overcome managerial diseconomies of scale. It has even transformed the nature of the R\&D process, the nature of their products, as well as the relationship of the systems integrator firms with their supply chain and with their customers. The pace of the ICT revolution is accelerating with the advent of cloud computing, Artificial Intelligence, Machine Learning and the Internet of Things.

The modern ICT revolution began in the 1970s. Intel was founded in 1968, and in 1971 it produced the world's first commercial microprocessor chip. In 1973, Hewlett Packard produced the first desktop micro-computer with a keyboard and mouse. In 1977, Apple launched the first mass-market ready-assembled personal computer. The sales of PCs reached 71 million in 1996, soaring to 366 million in 2011.

The ICT revolution accelerated with the emergence of the World Wide Web and the modern Internet in the mid-1990s, but its penetration has greatly increased in recent years: the number of Internet users increased from 390 million (16\% of the world population) in 2000 to 4.6 billion (59\% of the world population) in 2020 .

The share of telecommunications information carried over the Internet increased from $1 \%$ in 1993 to $51 \%$ in 2000, climbing to $97 \%$ in 2007.

Easily usable search engines began to emerge at the end of the 1990s: Yahoo was established in 1995 and Google in 1998. The combination of the PC, the World Wide Web and the web browser for access to the internet transformed a significant fraction of modern life, both business and personal.

The first mass-market mobile phone was produced by Nokia in 1992 . The first mass-market smartphone was launched by Apple (the iPhone) in 2007.

E-commerce took off in the new millennium: Amazon's revenues increased from USD 2.4 billion in 2004 to USD 34 billion in 2010, before accelerating to USD 281 billion in 2019.

Social media hardly existed before 2000. Facebook was founded in 2004 and YouTube in 2005. The number of 'active users' of social media increased from 970 million in 2010 to 3.8 billion in 2020. In July 2019, Facebook and YouTube both had over 2 billion 'active users'.

Servers have been a vital part of information technology networks throughout the modern information technology era. Advances in software and semi-conductors have combined with new generations of servers to produce a new era of information technology.

Clusters of private servers for local clients within firms and institutions have been increasingly superseded by public 'cloud' computing, based on giant data centres distributed across the world, connected by 'dark fibre' submarine and terrestrial communication networks.

Cloud computing services for data storage and analytics are paid for on a 'per service' (IaaS, PaaS and SaaS). ${ }^{1}$ Revenues from cloud computing have increased

\footnotetext{
${ }^{1}$ 'Infrastructure as a Service', 'Platform as a Service' and 'Software as a Service'.
} 
from less than USD 6 billion in 2008 to over USD 200 billion in 2019. Development of the 'cloud' has been greatly accelerated by the development of the 'Internet of Things' (IoT), with a huge expansion of embedded semi-conductors in almost every piece of complex machinery, from in-car infotainment systems to refrigerators. The global market for semi-conductors increased from USD 340 billion in 2015 to USD 440 billion in 2019, and is predicted to reach USD 650 billion in 2025. Advances in machine learning and artificial intelligence are likely to stimulate even further development of cloud computing.

The ICT sector has been at the leading edge of innovation in the recent era and it will be even more important in the years ahead. It is the sector in which by far the greatest amount is spent on R\&D, amounting to over two-fifths of total R\&D spending by the world's top 2,500 companies. ${ }^{2}$ Firms with their headquarters in the USA account for $55 \%$ of total R\&D spending on ICT hardware and software by G2500 companies. The dominance of American firms is especially noticeable in software and services: they account for $72 \%$ of total R\&D spending in this sector by G2500 firms.

The ICT industry has been characterized by a high number of mergers and acquisitions, which has contributed to a high level of industrial concentration in the industry, which is heavily research-intensive. In 2018/2019, R\&D spending in the ICT hardware and equipment sector amounted to $8.4 \%$ of net sales revenue and in the computer software and services sector, it amounted to $10.8 \%$ of sales revenue.

The ICT sector has evolved at tremendous speed. The innovations made by scientists and engineers in the firms within this broad sector have transformed the modern world, driven by high levels of R\&D spending and ferocious oligopolistic competition from the top to the bottom of the ICT value chain. Since the introduction of the semi-conductor and the PC, a wide array of new sectors have emerged within the ICT industry, but within each sector, an oligopoly has rapidly developed.

\section{Computer Software and Services}

There are 321 firms on the G2500 list that are in the computer software and services sector, of which the top 20 firms account for $67 \%$ of R\&D spending and $70 \%$ of sales revenue.

Microsoft has maintained its dominant position in PC operating systems, which it attained early on, for many years.

In the Enterprise Resource Planning (ERP) sector, the top five firms account for around one-half of the global market. With the exception of China, Google established its dominant position among the various search engines, while Facebook established a dominant position in social media, which they have maintained since then. Google

\footnotetext{
${ }^{2}$ The G2500 companies are the world's top 2500 companies ranked by R\&D spending. They account for around $90 \%$ of global corporate spending on R\&D (EU, 2019, The 2019 EU Industrial R\&D Investment Scoreboard, Brussels: EU).
} 
and Facebook account for over one-half of global digital advertising revenue. Google (Android) has around three-quarters of the global market for smartphone operating systems.

Cloud computing has grown rapidly over the past five years and is the foundation of the Internet of Things. Three super-large firms-Amazon, Microsoft and AlphabetGoogle - have leveraged their dominant position in other parts of the digital world to establish an early lead in cloud computing software and services. These three behemoths account for $38 \%$ of the total R\&D spending and $34 \%$ of the net sales revenue for the 321 firms in the G2500 ICT software and services sector. Collectively, they account for almost $60 \%$ of global revenue from software services for the public cloud.

The customers for the giant cloud companies' services are drawn from a wide array of sectors, including financial services, automobiles, energy systems, pharmaceuticals, health care, media and entertainment, retail, hospitality, manufacturing and government. They provide on-demand data storage, data analysis and machine learning for a wide array of sectors as well as on-demand cloud services for consumers, which means that they can avoid investing in their own "private cloud', which may operate at less than full capacity. Their customers benefit from state-of-the-art network infrastructure purchased by the giants' cloud computing companies.

The vast size of their network means that they can acquire equipment, which includes servers, routers and switches, more cheaply than small-scale private cloud systems. They play a vital role within the 'Internet of Things' that connects embedded semi-conductors across a wide array of machines. They also invest heavily in data security, including the security advantage of their closed-loop global dark fibre networks. They require a network of routers and switches to link the centres together, and need huge amounts of electricity to keep the server farms cool. It is estimated that $50 \%$ of the electricity used by data centres is devoted to keeping them cool.

\subsection{Amazon}

Amazon's AWS (Amazon Web Services) division increased its revenues from USD 1.5 billion in 2010 to USD 36 billion in 2019. By 2019, it accounted for around onethird of global revenues from cloud services. The rapid growth of Amazon's web services division was greatly helped by Amazon's leading position in e-commerce outside China and the consequent rapid growth of its revenues. Information technology is centrally important for Amazon's vast e-commerce business, including third-party sales. Amazon's revenues increased from USD 34 billion in 2010 to USD 280 billion in 2019. Its R\&D investment increased alongside its increased sales revenue, rising from USD 1.7 billion in 2010 to USD 35.9 billion in 2019, by which point Amazon was by far the largest global company in the world in terms of the size of its R\&D budget. Amazon has invested a significant proportion of its revenue in building data centres across the world: it has eight in the Americas, six in 
Europe/Middle East and eight in Asia-Pacific. Each centre consists of thousands of servers and Amazon's global system contains a total of 1.4 million servers.

\subsection{Microsoft}

Microsoft's cloud business is built on the foundation of Microsoft's long-held dominance of PC software, in which it still has around three-quarters of the global market. Microsoft's total revenues increased from USD 94 billion in 2015 to USD 126 billion in 2019. Microsoft consistently invests around $13 \%$ of its revenues in R\&D, which has supported its powerful competitive position in cloud services, the revenues of which are the fastest-growing part of Microsoft's revenue stream. Microsoft's total revenues from the 'Intelligent Cloud' reached USD 40 billion, within which cloud services made up USD 33 billion. Microsoft Azure, Microsoft's cloud service subsidiary, benefits from Microsoft's global network of data centres, including 14 in the Americas, eight in Europe and 15 in Asia-Pacific. Microsoft owns and operates its own Wide Area Network (WAN) of 'dark fibre' which connects its data centres with each other. During 2017-2020, Microsoft increased its WAN network seven-fold. It claims that its Software-Defined Network (SDN) is the 'fastest network of any in the public cloud'.

\subsection{Alphabet (Google)}

Google's Chrome search engine is the foundation of Alphabet's business. Outside China, Google's search engine 'dominates the market in all countries on any device, whether desktop, mobile or tablet', with a market share of over 90\%. As Alphabet's revenue has grown rapidly, it has maintained a high rate of investment in R\&D. Between 2014 and 2019, Alphabet's revenues increased from USD 66 billion to USD 161 billion, over $80 \%$ of which comes from Google Search, and investment in R\&D grew from USD 10 billion to USD 26 billion. Google Cloud software services division is linked closely to Google's global network of data centres: it has eight centres in the Americas, six in Europe and seven in Asia-Pacific. Google's public cloud software business has evolved out of its existing ICT-based businesses, including its Google Chrome search engine, Android operating system for smartphones, which it acquired in 2005, YouTube, which it acquired in 2006, Gmail and Google Maps. Google's global infrastructure serves six billion hours of YouTube video per month and provides data storage for one billion Gmail users. 


\subsection{Other Cloud Competitors}

Although these three behemoths have dominated the early phase of cloud computing, they face fierce rivalry from competitors who already have a strong position in the ICT industry. For example, IBM has a powerful platform from which to attack the market in cloud computing. IBM maintains a long-held monopoly over mainframe computers, which are still a significant source of revenue and profits. It also has a leading position in the server market. However, despite its continued strength in key components in the computer hardware market, IBM has made a long-term transition away from hardware. By 2019, two-thirds of its revenues came from software and services. IBM systems service $90 \%$ of the world's credit card transactions, and over four-fifths of the world's telecom companies are its customers. Since 2012, IBM has invested over USD 30 billion in capital expenditures and USD 45 billion in R\&D, much of it cloud-related. In 2019, it completed its acquisition of Red Hat to the tune of USD 34 billion in order to advance its competitive position in cloud computing.

\section{Technology Hardware}

There are 477 firms in the G2500 data set from the technology hardware sector. Of these, the top 20 firms account for $51 \%$ of R\&D spending and $66 \%$ of sales revenue.

Two firms (Samsung and Apple) account for three-fifths of the global market for smartphones (by revenue).

Servers are the workhorses of the whole ICT system, including the private and public cloud. Half a dozen firms, led by HPE and Dell, dominate the global server market.

In advanced TVs, Samsung alone accounts for over one-half of the global market (by sales revenue).

In telecom equipment, Huawei alone accounts for around one-third of the global market and the top five firms account for two-thirds. The value chain of these industries is also highly consolidated. One firm (Cisco) accounts for around one-half of the global market for telecoms routers and switches.

The semi-conductor sector also is highly concentrated. Five firms account for about one-half of the whole market, but levels of industrial concentration are even higher in most sub-sectors of the chip industry. Intel accounts for around threequarters of the global market for PC microprocessors. Two firms (Qualcomm and Apple) account for three-fifths of smartphone processors. One firm (Samsung) accounts for almost one-half of the global market for DRAM chips and a third of the market for NAND chips. Five firms account for around three-fifths of the global market for Wi-Fi chips. Five firms account for one-half of auto semi-conductors. Moreover, four firms account for two-thirds of the global market for semi-conductor equipment, which is a vital part of the innovation process in the semi-conductor industry. 
The technology hardware sector embraces a wide range of sub-sectors in terms of their R\&D intensity. PCs, printers and servers typically involve relatively low R\&D intensity. Leading technology hardware companies from the first generation of the ICT revolution, such as IBM and HP, have divested their PC and low-end server divisions in order to focus on other parts of the ICT industry, which have higher margins and profitability. ${ }^{3}$

Smartphones and tablets involve the medium intensity of $R \& D$ spending. However, they require high innovation skills in terms of product design and customer understanding. They also require the capability of integrating sophisticated systems. Manufacturing these products requires complex value chains across the world as well as a wide array of sub-systems and components, including software, semi-conductors, screens, batteries and camera lenses. This drive also requires considerable investments in marketing and branding.

Telecom equipment typically requires a high degree of R\&D intensity. Telecom equipment is typically customer-specific and innovation needs to closely integrate design, manufacturing and customer knowledge. ${ }^{4}$ The brand image and reputation of telecom equipment companies are strongly affected by the level and quality of customer support they provide after the sale has been completed.

Within the ITC hardware industry, semi-conductors are known for the intense research carried out to drive innovation. Of the top 30 ICT hardware firms, half are specialist semi-conductor manufacturers. However, besides making complex electronic and telecom equipment, Samsung, Apple and Huawei also are significant semiconductor producers. ${ }^{5}$ In 2018, Samsung's revenues from semi-conductors were over USD 60 billion, making it the world's second-largest chip-maker. A significant share of its huge R\&D budget of EUR 15 billion (2018) is devoted to driving technological capabilities in DRAM and NAND chips. In 2018, Samsung's semi-conductor division accounted for over three-quarters of its total profits. If we include the semiconductor equipment sector, then 22 out of the top 30 ICT hardware companies in terms of R\&D spending are in the semi-conductor sector, either as pure-play chip makers or companies with large semi-conductor sub-divisions. Semi-conductors are a crucial part of the whole ICT industry and the sector has been at the centre of the transformation of the modern world since the 1980s, but it will become even more important as the transition to the Internet of Things, Machine Learning and Artificial Intelligence continues. At the core of this 'connected world' will be 'hundreds of billions' of sensors and smart devices, which will result in a huge increase in the amount of data that will be generated, transmitted, stored, processed and analysed.

\footnotetext{
${ }^{3}$ Hewlett Packard was one of the earliest pioneers of the ICT revolution. In 2015, it divided into two: HP, which focused on low R\&D intensity PCs and printers, and HPE, which focused on high-end servers and other cloud-related technologies.

${ }^{4}$ A key part of Huawei's competitive success can be attributed to the mindset revolution produced by Huawei's Chairman Ren Zhengfei. Around the year 2000, he engaged a team of consultants from IBM at high cost to transform the thinking of Huawei's R\&D department away from a narrowly 'engineering' approach and focus on the individual customer's particular needs. In Ren's view, Huawei needed to 'cut its Chinese feet to fit American shoes'.

${ }^{5}$ Huawei's subsidiary, Hisileon is a semi-conductor maker.
} 


\section{Conclusion}

The role of Huawei in the Internet of Things has been one of the most fiercely argued issues in international relations in recent years.

In 2012, I described the rise of Huawei in my book Is China Buying The World? (Polity Press). The following excerpts from the book might provide some insight into Huawei, and in turn the degree of Chinese influence in ICT around the world.

In almost every discussion about China's 'catch-up' at the level of the firm, the case of Huawei arises. It has advanced from a minnow in the highly concentrated global telecom equipment industry to a giant firm with revenues in 2010 of $\$ 27.1$ billion and an operating profit of over $\$ 4.3$ billion. In the late 1990s Huawei comprehensively re-engineered the company, engaging IBM at great expense to lead the transformation from a technology-based to a customer-based approach. The reprocess was so painful that its CEO likened it to "cutting our feet to fit American shoes'. Huawei's foreign sales grew from \$100 million in 1999 to almost $\$ 18$ billion in 2010. Although its sales in developing economies are far greater than those in high-income countries, Huawei has made significant inroads into markets in the latter, especially in Europe. In 2005 it was certified as a qualified supplier to both BT and Vodafone, which required it to submit to the deepest scrutiny of its products and processes and all aspects of its performance, including not just technical issues but also its compliance with internationally accepted practices in terms of corporate social responsibility. Among large Chinese firms Huawei is unique in having met the most severe standards of global competition among customers in the high-income countries: it stands alone in being 'inside us'. It is unusual among large Chinese firms in terms of the continuity of its top management, its focus on core business, the high share of revenue allocated to $R \& D$, the large share of its employees engaged in $R \& D$, the large share of foreign workers among its employees, the open and transparent system of organization and remuneration of its workforce, the intellectual and physical attractiveness of the work environment, and the internationalization of its culture, including the use of English throughout the upper reaches of the company.

The recent attempts to thwart the international rise of Huawei, particularly in 5G development outside of China, is nothing new. This is evidence from what I wrote in 2012 in Is China Buying The World?.

A succession of possible international acquisitions by Huawei were all abandoned. In 2005 it was rumoured that it was in negotiations to acquire Marconi, the venerable but loss-making UK telecoms equipment maker. This prompted intense discussion in the UK mass media and rumours that the deal would be referred to the US government's Committee on Foreign Investment in the United States (CFIUS). Huawei made no formal offer to acquire Marconi, and eventually it was sold to Ericsson for $\$ 2.1$ billion. In 2010 Huawei made a bid to acquire the tiny niche telecoms software company 3Leaf for $\$ 2$ million, a minuscule transaction in global terms. The deal was blocked by CFIUS on national security grounds. An alternative to full-scale takeover is the acquisition by Chinese companies of substantial minority shares in leading Western companies. In 2007-8 it was proposed that Huawei would acquire 3Com, the US telecoms equipment company, jointly with the US private equity firm Bain Capital. Despite the fact that Huawei would own only a small minority share, and despite the fact that 3 Com is a relatively small company, the proposal led to an intense US media furore focusing on Huawei's 'threat to US national security', and the case was referred to CFIUS. Before a formal ruling was reached, the acquisition offer was withdrawn by Bain and Huawei. In 2010 HP acquired 3Com for $\$ 2.2$ billion. 
Huawei is China's only high technology company with a significant global market share outside China. The company has been relentless in expansion in telecom equipment and is widely accepted to be the tech leader in $5 \mathrm{G}$ telecom equipment. Its global market share in $5 \mathrm{G}$ transmissions equipment may be as high as $35 \%$, more than the combined share of its closest competitors, Nokia and Ericsson.

However, the role of Huawei needs to be analysed in relation to the overall structure of the fast-developing Internet of Things.

Each segment of this vast architecture has become highly consolidated, with a few companies, almost all from high-income countries, which dominate each part of the architecture. The Internet of Things is made up of a massive overarching ICT architecture that requires a huge network of base stations as well as a global network of 'dark fibre', a widely distributed global network of data centres full of servers, a cloud computing software system (notably IaaS, PaaS), a global array of billions of smartphones, semi-conductors and software within the smartphones, and hundreds of billions of semi-conductors embedded with 'connected devices'.

Huawei has a relatively minor position in operating the global structure of massive data centres and dark fibre and secure global networks that connect them. Huawei is also relatively negligible in other areas, including its global market share in the server hardware that comprises cores of data centres, its role in the cloud computing software services as well as its market share of the various markets for semi-conductors, which are embedded in countless millions of 'connected devices'. Huawei also does not have much influence in global search engine and browser systems that produces a vast amount of data.

Huawei has developed a significant, but far from dominant market share in smartphone handsets, but the core operating systems inside almost all smartphones, including Huawei's devices, are dominated by Google-Android. The wide array of semi-conductors and software systems inside the world's billions of smartphones are also dominated by a small group of high technology companies with their headquarters in the high-income countries, led by the US.

Global security in the Internet of Things needs to be considered in terms of not just a single part of the architecture, but rather, in terms of the comprehensive structure of global data transmission, storage and analysis. Huawei has a significant role within one segment of that structure. However, within the whole structure, it has a small role. So far, the entire structure is dominated by firms from high-income countries, especially the US.

Peter Nolan is the Founding Director of Cambridge University's Centre of Development Studies and the Director of Jesus College's China Centre. He is the Director of the Chinese Executive Leadership Programme (CELP), which each year brings chief executives from China's largest firms to the University of Cambridge to attend training programmes taught by academics and the leaders of international firms. The Financial Times commented: "Nolan knows more about Chinese companies and their international competition than anyone else on earth, including in China". Peter Nolan has researched, written and taught on a wide range of issues in economic development, globalisation and the transition of former planned economies. 
Open Access This chapter is licensed under the terms of the Creative Commons AttributionNonCommercial-NoDerivatives 4.0 International License (http://creativecommons.org/licenses/bync-nd/4.0/), which permits any noncommercial use, sharing, distribution and reproduction in any medium or format, as long as you give appropriate credit to the original author(s) and the source, provide a link to the Creative Commons license and indicate if you modified the licensed material. You do not have permission under this license to share adapted material derived from this chapter or parts of it.

The images or other third party material in this chapter are included in the chapter's Creative Commons license, unless indicated otherwise in a credit line to the material. If material is not included in the chapter's Creative Commons license and your intended use is not permitted by statutory regulation or exceeds the permitted use, you will need to obtain permission directly from the copyright holder.

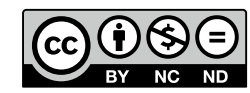

